\title{
COVID-19 in Egyptian Children: A Multicenter Study
}

\author{
Amin Abdel Baki ${ }^{10}$ Samy Zaky ${ }^{2}$ Hossam Hosny ${ }^{3}$ Gehan Elassal ${ }^{4}$ He $^{(0 k r a m ~ A b d e l b a r y}{ }^{5}$ \\ Ahmad Said ${ }^{5}$ Ehab Kamal ${ }^{6 \odot}$ Noha Asem ${ }^{7,8}$ Hamdy Ibrahim ${ }^{1}$ Alaa Eid $^{8}$ Wagdy Amin ${ }^{8}$ \\ Mohamed El Badry ${ }^{9}$ Abdelmajeed Mossa ${ }^{9}$ Fathiya El-Raey ${ }^{10}$ Shazly Baghdady ${ }^{11}$ \\ Marwa Tahoon ${ }^{12}$ Mohamed Hassany ${ }^{1,8}$ Hala Zaid $^{8}$
}

${ }^{1}$ Department of Hepatogastroenterology and Infectious Diseases, National Hepatology and Tropical Medicine Research Institute, Cairo, Egypt

2 Department of Hepatogastroenterology and Infectious Diseases, Al-Azhar University, Cairo, Egypt

${ }^{3}$ Department of Pulmonary Medicine, Faculty of Medicine, Cairo University, Cairo, Egypt

${ }^{4}$ Department of Pulmonary Medicine, Faculty of Medicine, Ain Shams University, Cairo, Egypt

${ }^{5}$ Department of Critical Care, Faculty of Medicine, Cairo University, Cairo, Egypt

${ }^{6}$ Division of Tropical Medicine Medical Research, National Research Centre, Giza, Egypt

${ }^{7}$ Department of Public Health and Community Medicine, Faculty of Medicine, Cairo University, Cairo, Egypt

8 Ministry of Health and Population, Egypt
Address for correspondence Mohamed Hassany, MD, Department of Hepatogastroenterology and Infectious Diseases, National

Hepatology and Tropical Medicine Research Institute, 10 Kasr Al Aini

Street, Cairo 11441, Egypt (e-mail: mohamadhassany@yahoo.com).

${ }^{9}$ Tropical Medicine and Gastroenterology Department, Aswan University, Aswan, Egypt

10 Hepatogastroenterology and Infectious Diseases Department, Al-Azhar University, Damietta, Egypt,

${ }^{11}$ Department of Chest, Faculty of Medicine, Aswan University, Aswan, Egypt,

12 Department of Epidemiology and Preventive Medicine, National Liver Institute, Menoufia University, Menofia Governorate, Egypt

J Pediatr Infect Dis 2021;16:57-61.

\begin{abstract}
Keywords

- COVID-19

- Egypt

- children

Objective The novel coronavirus disease 2019 (COVID-19) has made its worldwide spread since its outbreak in December 2019. Limited information is available about the epidemiology and clinical characteristics of COVID-19, especially in Africa and Egypt. Methods We aimed to study the clinical and epidemiological characteristics of COVID-19 in Egyptian children. This is an observational retrospective cohort study performed at two specialized COVID-19 isolation hospitals in Egypt. All admitted COVID-19 pediatric patients between March 20, 2020, and May 1, 2020, were included in the study.

Discussion This study included 40 COVID-19 confirmed cases (mean age, 9.4 years), $67.5 \%$ were male, $85 \%$ were asymptomatic, and $15 \%$ had mild symptoms. There were no confirmed severe or critically ill cases among the patients.

Conclusion COVID-19 runs in a benign course in Egyptian children with no mortality and no significant morbidity.
\end{abstract}

\section{Introduction}

By the end of 2019, severe acute respiratory syndromecoronavirus-2 (SARS-CoV-2), originated in Wuhan City in Hubei Province of China, was recognized as an etiology for a

received

July 11,2020

accepted after revision

November 29, 2020

published online

February 1, 2021 series of severe cases of pneumonia. ${ }^{1}$ The disease had been commonly termed as novel coronavirus disease 2019 (COVID-19), and due to its worldwide spread, it was classified by the World Health Organization (WHO) as a global

(c) 2021. Thieme. All rights reserved. Georg Thieme Verlag KG, Rüdigerstraße 14,

70469 Stuttgart, Germany
DOI https://doi.org/ 10.1055/s-0040-1722284. ISSN 1305-7707. 
pandemic on March 11, 2020. ${ }^{2}$ The first COVID-19 confirmed case in Egypt was announced by the Ministry of Health and Population (MoHP) on February 14, 2020, ${ }^{2}$ and as of May 26, 2020, there have been 17,967 confirmed cases and 783 deaths. $^{3}$ In children, the first positive COVID-19 case was reported on January 20, 2020, in China. ${ }^{4}$ Infection with SARSCoV-2 in children is less common than in adults, and often they have a mild form of the disease and a lower mortality rate $^{5}$; children account for an estimated 1 to $5 \%$ of patients diagnosed with COVID-19. ${ }^{6}$

Studies conducted on the Chinese population helped confirm that the most common source for the infection in children was the exposure to an infected household family member. $^{7}$

The clinical manifestations of COVID-19 in children are similar to other viral respiratory tract infections, namely, fever, cough, shortness of breath, sore throat, diarrhea, nausea, vomiting, anorexia, and myalgia. ${ }^{8}$ Some studies also reported that many confirmed COVID-19 children are asymptomatic at presentation. ${ }^{9}$ However, in Africa, there is limited available data on COVID-19, and to the best of our knowledge, there have been no published data on COVID-19 in African children so far; in the light of this, the current study aimed at identifying the course of COVID-19 in Egyptian children in two specialized isolation hospitals.

\section{Materials and Methods}

This was an observational retrospective cohort study at two specialized isolation hospitals for COVID-19 in Aswan (Aswan Specialized Hospital, south of Egypt) and Damietta (Damietta Specialized Hospital, north of Egypt) governorates in Egypt. The MoHP in Egypt assigned particular hospitals in each governorate for triage of suspected COVID-19 cases and isolation of the confirmed cases. We included all children (1-18 years) with laboratory-confirmed COVID-19 by reverse transcription polymerase chain reaction (RT-PCR; VIASURE [CerTest Biotec; Zaragoza, Spain] and PerkinElmer [PerkinElmer Inc.; Turku, Finland]) of samples obtained by nasopharyngeal swabs and who were admitted to these two isolation hospitals in the period between March 20, 2020, and May 1, 2020; patients were managed according to the released national guidelines. ${ }^{3}$

Demographic data, medical history, clinical manifestations, laboratory data, radiological characteristics, drugs that were given during the illness, and the outcome of the disease were all obtained from the patient's medical record. For the epidemiological history, we focused on travel and vaccination history, residence in epidemic areas or areas with clusters and outbreak, and history of contact with confirmed COVID-19 patients within the past 14 days. According to the Egyptian protocol for the treatment of COVID-19 and its updated versions thereof were declared by the supreme scientific committee for management of COVID-19 (affiliated to MoHP), nasopharyngeal swab samples were collected by a trained laboratory technician from patients with suspected SARS-CoV-2 infection; the samples were then processed for RNA extraction and those who tested positive for COVID-19 were admitted and started treatment. On day 5 of admission, repetition of the PCR samples was done to check for viral clearance. Patients were considered cured if they became clinically cured after testing negative for the infection after two consecutive PCR tests. Laboratory tests included complete blood count and serum biochemical tests, liver and kidney functions, and coagulation profile. Radiological investigations included chest X-ray and chest computed tomography (CT) scans. Two experienced radiologists reviewed the radiological diagnoses. Treatment of the patients included supportive measures, antipyretics, close monitoring, and fluid (conservative) oseltamivir (dose in children less than $40 \mathrm{~kg}$ body weight: $6.5 \mathrm{mg} / \mathrm{kg}$, then $3.25 \mathrm{mg} / \mathrm{kg} / 12$ hours). Varying treatment dosages were administered, depending on the age group: (1) children less than 8 years received only oseltamivir (dose is according to age and body weight), data were limited and the medication was not recommended unless the situation was deemed critical for patients $<3$ months, children $>3$ months received $12 \mathrm{mg}$ PO q12 hours for 5 days, children 3 to 5 months received $20 \mathrm{mg}$ PO q12 hours for 5 days, and children 6 to 11 months received $25 \mathrm{mg}$ PO q12 hours for 5 days; (2) if 1 year or older, the dose was variable according to child's weight: for $15 \mathrm{~kg}$ or less, the dose was $30-\mathrm{mg}$ twice a day, for $>15$ to $23 \mathrm{~kg}$, the dose was $45-\mathrm{mg}$ twice a day, for $>23$ to $40 \mathrm{~kg}$, the dose was $60-\mathrm{mg}$ twice a day, and for $>40 \mathrm{~kg}$, the dose was 75-mg twice a day for 5 days; (3) those above 8 years received a 5-day course combination of oseltamivir and hydroxychloroquine. For the clinical outcomes, the patient was declared cured when the symptoms of the infection were absent for at least 3 consecutive days without taking antipyretics and fulfilling two consecutive negative results of RT-PCR with a 48-hour interval. On the other hand, negative outcomes were referred to as disease progression to critical illness or, at worse, death. Only patients who achieved cured outcomes were discharged from the hospital.

Data were collected, coded, revised, and entered into the Statistical Package for Social Science (IBM SPSS) version 20. The data were presented as numbers and percentages for the qualitative data, mean, and standard deviations (SDs); as ranges for the quantitative data with parametric distribution and as median with interquartile range (IQR) for the quantitative data with the nonparametric distribution.

\section{Results}

Between March 20 and May 1, 2020, 532 COVID-19 cases in total were admitted to Aswan and Damietta specialized hospitals in Egypt-40 (7.5\%) of these cases were children (8 months-17 years; mean age, 9.4 years), and 27 (67.5\%) of the 40 patients were male (-Table 1 ). The identified common source of infection for all the pediatric patients was being in close contact with a COVID-19-positive household family member. All patients had no underlying chronic diseases at presentation, and all of them received their compulsory vaccination according to the national program guidelines. However, at the time of admission, 34 (85\%) of the 40 patients were asymptomatic and 6 (15\%) had mild 
Table 1 Demographic and clinical data of the studied patients

\begin{tabular}{|l|l|l|l|}
\hline \multicolumn{2}{|c|}{ Demographic and clinical data } & $\boldsymbol{n}$ & $\%$ \\
\hline Age $(\mathrm{y})$ & $\leq 8$ & 17 & 42.5 \\
\hline & $>8$ & 23 & 57.5 \\
\hline Gender & Male & 27 & 67.5 \\
\hline & Female & 13 & 32.5 \\
\hline Symptoms & Asymptomatic & 34 & 85.0 \\
\hline & Cough & 1 & \multirow{2}{*}{15.0} \\
\hline & Fatigue & 1 & \multirow{2}{*}{} \\
\cline { 1 - 3 } & Fever & 3 & \multirow{2}{|l|}{} \\
\hline & Sore throat & 1 & \\
\cline { 1 - 3 } & &
\end{tabular}

symptoms, as shown in -Table 1. There were no severe or critically ill cases. No abnormal radiological findings were seen in the chest X-ray or CT scans for all patients. All patients had unremarkable laboratory findings at the time of admission, as shown in - Table 2.

At treatment, 17 patients (42.5\%) received oseltamivir and 23 patients (57.5\%) received combinations of hydroxychloroquine and oseltamivir for 5 days. Clinical outcomes reported no mortality or critical illness; all the patients were defined as cured. The number of days required to achieve a cured outcome are shown in - Fig. 1, with a mean $\pm($ SD) and range corresponding to $9.5 \pm(3.4)$ and 5 to 17 days, respectively. The relation between the number of days required for cure and different parameters (age, gender, symptoms, and antiviral treatment) are described in - Table 3. The length of hospital stay was from 7 to 19 days, and most of the patients (75\%) stayed more than 10 days in the hospitals.

Table 2 Baseline laboratory data of the studied patients

\begin{tabular}{|l|l|l|}
\hline Parameters & Mean \pm SD & Range \\
\hline Hemoglobin & $11.9 \pm 0.87$ & $10.5-13.5 \mathrm{~g} / \mathrm{dL}$ \\
\hline Lymphocyte & $26.3 \% \pm 7.4$ & $18-45 \%$ \\
\hline Platelets count & $241.5 \pm 71.6$ & $125-452 \times 10^{9} / \mathrm{L}$ \\
\hline $\begin{array}{l}\text { White blood } \\
\text { cells }\end{array}$ & $6.2 \pm 1.9$ & $3.6-11 \times 10^{9} / \mathrm{L}$ \\
\hline $\begin{array}{l}\text { Prothrombin } \\
\text { time }\end{array}$ & $13.6 \pm 0.48$ & $13.0-14.5$ seconds \\
\hline $\begin{array}{l}\text { International } \\
\text { normalization } \\
\text { ratio }\end{array}$ & $1.1 \pm 0.086$ & $1.0-1.2$ \\
\hline Blood urea & $28.9 \pm 6.3$ & $20-40 \mathrm{mg} / \mathrm{dL}$ \\
\hline $\begin{array}{l}\text { Serum } \\
\text { creatinine }\end{array}$ & $0.76 \pm 0.16$ & $0.4-1.1 \mathrm{mg} / \mathrm{dL}$ \\
\hline ALT & $24.9 \pm 7.6$ & $14-41 \mathrm{U} / \mathrm{L}$ \\
\hline AST & $25.9 \pm 8.4$ & $14-40 \mathrm{U} / \mathrm{L}$ \\
\hline
\end{tabular}

Abbreviations: ALT, alanine transaminase; AST, aspartate transaminase; SD, standard deviation.

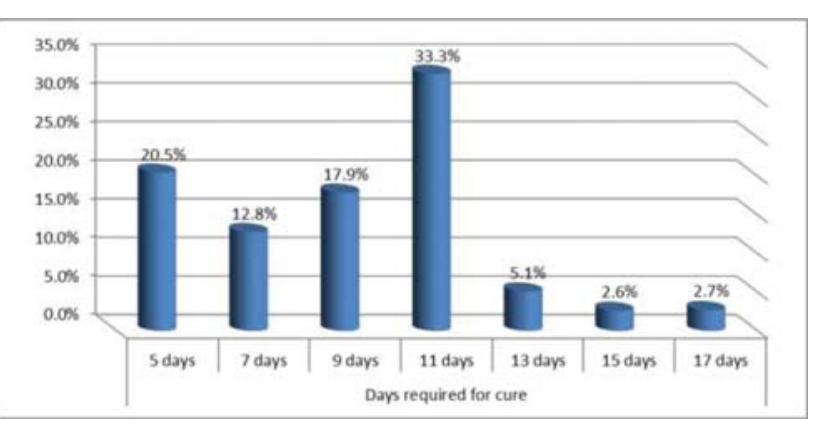

Fig. 1 Days required for patients' cure.

\section{Discussion}

Since the diagnosis of the first case of COVID-19 in Egypt, also considered the first declared case in Africa, ${ }^{2}$ thousands of cases in the different age groups were reported. Moreover, a critical observation was made concerning the pediatric population, there was a smaller number of infected children compared with adults, and their vulnerability in developing significant morbidity and mortality was lower ${ }^{5}$-this certainly creates a fertile area of research about the possible causes of this infection in the general population.

Among the confirmed positive cases in Egypt till May 1, $2020,7.5 \%$ were children, which is a relatively high percentage compared with those in China (2.2\%), Italy (1.2\%), and Spain (2\%). ${ }^{9-11}$ The high percentage of the infected children is relative to the higher distribution of children in the population pyramid, which reached the $42.4 \%$ of the population in $\leq 19$-year-old children in $2019,{ }^{12}$ in addition to the active tracing and testing of all household and family members of the confirmed cases.

In line with our study, recent studies also reported children having lower rates of SARS-CoV-2 infection than adults. In the light of this, it is hypothesized that the limited symptomatic cases in children make most of the children asymptomatic, neglecting them as targets of testing. Consequently, this led to a lower number of tested children worldwide compared with adults. ${ }^{6}$ It is thought that children might be protected against COVID-19 due to compulsory viral vaccinations given routinely in early childhood, ${ }^{13}$ which stimulates T-helper cells to secrete different cytokines that enhance the function of natural killer cells ${ }^{14}$; also, the children's weak immune system could not exaggerate the immune response against SARS-Cov-2 in contrast to adults ${ }^{15}$; moreover, the angiotensin-converting enzyme 2 (ACE2) gene expression in the nasal epithelium is lowest in younger children and increases with age. However, children may be less exposed to the infected community. ${ }^{16}$

Concerning the suggested mode of transmission, all cases got infected due to contact with confirmed COVID-19 household members, and it is similar to what had been reported from other studies conducted in China. ${ }^{4-8}$ At the time of presentation, $85 \%$ of patients were asymptomatic, while the rest of them had only mild symptoms, denoting that there was no need for hospital admission; only home isolation if socially applicable with advice to monitor symptoms. This 
Table 3 Relation between different parameters and days required for cure

\begin{tabular}{|c|c|c|}
\hline & $\begin{array}{l}\text { Days required } \\
\text { for cure (mean } \pm S D \text { ) }\end{array}$ & $p$-Value \\
\hline Age (y) & & \multirow[t]{2}{*}{0.816} \\
\hline $\begin{array}{l}\leq 8 \\
>8\end{array}$ & $\begin{array}{l}9.5 \pm 3.1 \\
9.5 \pm 3.7\end{array}$ & \\
\hline Gender & & \multirow[t]{3}{*}{$0.022^{a}$} \\
\hline Male & $8.7 \pm 3.5$ & \\
\hline Female & $11.2 \pm 2.5$ & \\
\hline Symptoms & & \multirow[t]{3}{*}{0.929} \\
\hline Asymptomatic & $9.8 \pm 3.2$ & \\
\hline Symptomatic & $9.5 \pm 3.5$ & \\
\hline Treatment & & \multirow[t]{3}{*}{0.977} \\
\hline Oseltamivir & $9.5 \pm 3.2$ & \\
\hline $\begin{array}{c}\text { Oseltamivir }+ \\
\text { hydroxychloroquine }\end{array}$ & $9.6 \pm 3.6$ & \\
\hline
\end{tabular}

Abbreviation: SD, standard deviation.

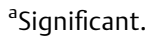

percentage of asymptomatic patients was considered to be higher when compared with the $13 \%$ in one study done in 2,000 Chinese pediatric patients. ${ }^{7}$ This finding could be explained by the early diagnosis in our cases, as they were tested because of their direct contact with infected household members and a small number of cases in our study.

Symptomatic cases were having mild symptoms. In our study, there were no severe or critical cases nor deaths; by that, it seems that COVID-19 in children is less severe than in adults. Similar findings were reported in studies from China ${ }^{17}$ and the United States. ${ }^{18}$

In our study, there were no significant laboratory findings; this is in line with the results reported by Henry and his colleagues which showed no significant effect of COVID-19 on complete blood count (CBC), liver enzymes, or serum creatinine in infected children. ${ }^{19}$ All our patients' radiological scans revealed unremarkable changes, in contrast to chest $\mathrm{X}$-rays of many pediatric patients showing pneumonic patches, and their CT scans had shown ground-glass opacities in many other studies. ${ }^{20-22}$ The mean \pm (SD) and range of the days required for the patients to achieve cured outcomes were $9.5 \pm(3.4)$ and 5.0 to 17.0 , respectively. All patients were discharged from the hospital after the second consecutive negative sample of RT-PCR at the time of the study; the discharge criteria were "test-based strategy." There was no significant relationship between the days required for cure and patients' age, symptoms, or antiviral treatment, but males needed fewer days to achieve cured outcomes compared with females. No data are available about the clinical impact of gender difference on COVID-19-infected children, yet. However, few available data in adults showed that being male is associated with higher morbidity and mortality independently of age. ${ }^{23}$

Hydroxychloroquine is one of the potential therapies used during the COVID-19 pandemic; it reduced viral replication in in vitro studies; however, animal and human studies showed conflicting results. ${ }^{24}$ Although the known side effects of chloroquine derivatives come from a long experience of its use in different clinical indications, ${ }^{25}$ there were no reported side effects in this study among patients who received hydroxychloroquine. Our results have not demonstrated any superiority of hydroxychloroquine use with oseltamivir for COVID-19 therapy compared with oseltamivir alone in children who presented a mild disease. Multicenter initial guidance on the use of antivirals for children with COVID-19/SARS-CoV-2 concluded that antiviral drugs are unnecessary for most of the children with COVID-19, and hydroxychloroquine could be considered when remdesivir is not available. ${ }^{26}$ Hydroxychloroquine use in children in the management of chronic diseases, for example, lupus, can be continued if there are no contraindications. All these findings support the benign behavior of COVID-19 in Egyptian children.

This study includes the following four limitations:

1. Small sample size and inability to do randomization.

2. Since the study is a retrospective cohort, and the data were obtained from the hospital records, no information was available about the size of the family and the housing conditions.

3. The study was conducted on data retrieved from isolation hospitals since the beginning of the pandemic, when no unified testing or treatment strategy was adopted; hence, testing, admission, and treatment of asymptotic children were the prevailed scenario.

4. PCR testing was done via a qualitative, not quantitative, assay, so no follow-up of viral load could be obtained.

\section{Conclusion}

Our study revealed a relatively high percentage of pediatric cases among admitted COVID-19 patients. Most of the cases were asymptomatic, and few of them had mild symptoms with no abnormal laboratory or radiological findings. In addition, no apparent benefit was confirmed adding hydroxychloroquine to the clinical management of pediatric patients with mild COVID-19. COVID-19 follows a benign course in Egyptian children with no progression to considerable morbidity or further mortality.

Conflict of Interest

None declared.

\section{References}

1 Wu P, Hao X, Lau EHY, et al. Real-time tentative assessment of the epidemiological characteristics of novel coronavirus infections in Wuhan, China, as at 22 January 2020. Euro Surveill 2020;25(03): 2000044

2 Hassany M, Abdel-Razek W, Asem N, AbdAllah M, Zaid H. Estimation of COVID-19 burden in Egypt. Lancet Infect Dis 2020;20(08): 896-897

3 The Egyptian Ministry of Health official report. 2020. Available at: http://www.mohp.gov.eg

4 Chan JFW, Yuan S, Kok KH, et al. A familial cluster of pneumonia associated with the 2019 novel coronavirus indicating person-to- 
person transmission: a study of a family cluster. Lancet 2020;395 (10223):514-523

5 Su L, Ma X, Yu H, et al. The different clinical characteristics of corona virus disease cases between children and their families in China - the character of children with COVID-19. Emerg Microbes Infect 2020;9(01):707-713

6 Ludvigsson JF. Systematic review of COVID-19 in children shows milder cases and a better prognosis than adults. Acta Paediatr 2020;109(06):1088-1095

7 Ji LN, Chao S, Wang YJ, et al. Clinical features of pediatric patients with COVID-19: a report of two family cluster cases. World J Pediatr 2020;16(03):267-270

8 Qiu H, Wu J, Hong L, Luo Y, Song Q Chen D. Clinical and epidemiological features of 36 children with coronavirus disease 2019 (COVID-19) in Zhejiang, China: an observational cohort study. Lancet Infect Dis 2020;20(06):689-696

9 Livingston E, Bucher K. Coronavirus Disease 2019 (COVID-19) in Italy. JAMA 2020;323(14):1335

$10 \mathrm{Wu}$ Z, McGoogan JM. Characteristics of and important lessons from the coronavirus Disease 2019 (COVID-19) outbreak in China: summary of a report of 72314 cases from the Chinese Center for Disease Control and Prevention. JAMA 2020;323(13): 1239-1242

11 Tagarro A, Epalza C, Santos M, et al. Screening and severity of coronavirus Disease 2019 (COVID-19) in children in Madrid, Spain. JAMA Pediatr 2020 (e-pub ahead of print). Doi: 10.1001/ jamapediatrics.2020.1346

12 Population Pyramids of the World from 1950 to 2100: Egypt 2019. Available at: https://www.populationpyramid.net/egypt/2019/. Accessed December 14, 2020

13 Salman S, Salem ML. Routine childhood immunization may protect against COVID-19. Med Hypotheses 2020;140:109689

14 Schouten LR, van Kaam AH, Kohse F, et al; MARS consortium. Agedependent differences in pulmonary host responses in ARDS: a prospective observational cohort study. Ann Intensive Care 2019;9(01):55
15 Chun Y, Do A, Grishina G, et al. Integrative study of the upper and lower airway microbiome and transcriptome in asthma. JCI Insight 2020;5(05):e133707

16 Bunyavanich S, Do A, Vicencio A. Nasal gene expression of angiotensin-converting Enzyme 2 in children and adults. JAMA 2020;323(23):2427-2429

17 Dong Y, Mo X, Hu Y, et al. Epidemiology of COVID-19 among children in China. Pediatrics 2020;145(06):e20200702

18 Bialek S, Boundy E, Bowen V, et al; CDC COVID-19 Response Team. Severe outcomes among patients with coronavirus disease 2019 (COVID-19)-United States. Morb Mortal Wkly Rep 2020;69(12): 343-346

19 Henry BM, Lippi G, Plebani M. Laboratory abnormalities in children with novel coronavirus disease 2019. Clin Chem Lab Med 2020;58(07):1135-1138

$20 \mathrm{Xu} \mathrm{Y,} \mathrm{Li} \mathrm{X,} \mathrm{Zhu} \mathrm{B,} \mathrm{et} \mathrm{al.} \mathrm{Characteristics} \mathrm{of} \mathrm{pediatric} \mathrm{SARS-CoV-2}$ infection and potential evidence for persistent fecal viral shedding. Nat Med 2020;26(04):502-505

21 Chen F, Liu ZS, Zhang FR, et al. [First case of severe childhood novel coronavirus pneumonia in China] (in Chinese). Zhonghua Er Ke Za Zhi 2020;58(00):E005

22 Feng K, Yun YX, Wang XF, et al. [Analysis of CT features of 15 children with 2019 novel coronavirus infection] (in Chinese). Zhonghua Er Ke Za Zhi 2020;58(00):E007

23 Jin JM, Bai P, He W, et al. Gender differences in patients with COVID19: focus on severity and mortality. Front Public Health 2020;8:152

24 Yao X, Ye F, Zhang M, et al. In vitro antiviral activity and projection of optimized dosing design of hydroxychloroquine for the treatment of severe acute respiratory syndrome coronavirus 2 (SARSCoV-2). Clin Infect Dis 2020;71(15):732-739

25 Juurlink DN. Safety considerations with chloroquine, hydroxychloroquine and azithromycin in the management of SARS-CoV-2 infection. CMAJ 2020;192(17):E450-E453

26 Chiotos K, Hayes M, Kimberlin DW, et al. Multicenter interim guidance on use of antivirals for children with COVID-19/SARSCoV-2. J Pediatr Infect Dis Soc 2021;10(01):34-48 